\title{
Association of breast-fed neonatal hyperbilirubinemia with UGT1A1 polymorphisms: 211G $>$ A (G71R) mutation becomes a risk factor under inadequate feeding
}

\author{
Hiroko Sato ${ }^{1}$, Toshihiko Uchida ${ }^{2}$, Kentaro Toyota ${ }^{1}$, Miyako Kanno ${ }^{1}$, Taeko Hashimoto ${ }^{1}$, Masashi Watanabe ${ }^{2}$, \\ Tomohiro Nakamura ${ }^{3}$, Gen Tamiya ${ }^{3}$, Kuraaki Aoki ${ }^{1}$ and Kiyoshi Hayasaka ${ }^{1}$
}

Breastfeeding jaundice is a well-known phenomenon, but its pathogenesis is still unclear. Increased production of bilirubin, impaired hepatic uptake and metabolism of bilirubin, and increased enterohepatic circulation of bilirubin account for most cases of pathological neonatal hyperbilirubinemia. We previously reported that $211 \mathrm{G}>\mathrm{A}(\mathrm{G} 71 \mathrm{R})$ mutation of the UGT1A1 gene is prevalent in East Asians and is associated with the development of neonatal hyperbilirubinemia. Recently, significant association of G71R mutation with hyperbilirubinemia in breast-fed neonates was reported. We enrolled 401 full-term Japanese infants, who were exclusively breast-fed without supplementation of formula before developing hyperbilirubinemia, and classified them into two groups based on the degree of maximal body weight loss during the neonatal period. We analyzed the sex, gestational age, delivery mode, body weight at birth, maximal body weight loss and genotypes of G71R and (TA) 7 polymorphic mutations of UGTIA1. Statistical analysis revealed that maximal body weight loss during the neonatal period is the only independent risk factor for the development of neonatal hyperbilirubinemia. The effect of G71R mutation on neonatal hyperbilirubinemia is significant in neonates with $5 \%$ or greater maximal body weight loss and its influence increases in parallel with the degree of maximal body weight loss. Our study indicates that G71R mutation is a risk factor for neonatal hyperbilirubinemia only in infants with inadequate breastfeeding and suggests that adequate breastfeeding may overcome the genetic predisposing factor, G71R mutation, for the development of neonatal hyperbilirubinemia. Journal of Human Genetics (2013) 58, 7-10; doi:10.1038/jhg.2012.116; published online 27 September 2012

Keywords: breastfeeding; Gilbert syndrome; neonatal hyperbilirubinemia; UGT1A1

\section{INTRODUCTION}

Unconjugated hyperbilirubinemia in neonates is a physiological and common phenomenon. Bilirubin production is increased in the neonate because of the larger erythrocyte volume, shortened erythrocyte lifespan, heme and heme precursors degraded from the fetal extramedullary hematopoietic tissue, and, possibly, increased turnover of cytochromes. ${ }^{1}$ The ability to conjugate bilirubin is also extremely low in neonates at term, being about $1 \%$ of adult values. ${ }^{2}$ Neonatal hyperbilirubinemia is also probably associated with other factors such as an immaturity of hepatic bilirubin uptake and intracellular bilirubin transport, and the increased enterohepatic circulation of bilirubin. The imbalance between the production and elimination of bilirubin leads to varying levels of hyperbilirubinemia in every neonate. The propensity toward neonatal hyperbilirubinemia in East Asians is well known. ${ }^{1}$ Association of mutation of the UGT1A1 gene,
$(\mathrm{TA})_{7}$ causing Gilbert syndrome, has been reported. ${ }^{3}$ In 1998 , we found that $211 \mathrm{G}>\mathrm{A}(\mathrm{G} 71 \mathrm{R})$ mutation of the UGT1A1 gene is very common among Japanese, Koreans and Chinese, and this mutation is associated with neonatal hyperbilirubinemia. ${ }^{4,5}$ Recently, the association of G71R mutation with neonatal hyperbilirubinemia was also confirmed in Asians $^{6}$ and was especially significant in breast-fed neonates. ${ }^{7,8}$ Regarding breastfeeding jaundice, appropriately known as breast-non-feeding jaundice, it has been considered to result from inadequate calorie intake. ${ }^{9}$

In the present paper, we studied the association of G71R and (TA $)_{7}$ mutations and hyperbilirubinemia in breast-fed Japanese neonates, and found that the maximal body weight loss during the neonatal period is an independent risk factor for the development of neonatal hyperbilirubinemia, and G71R mutation is a significant risk factor only in neonates with $5.0 \%$ or greater maximal body weight loss.

${ }^{1}$ Department of Pediatrics, Yamagata University School of Medicine, Yamagata, Japan; ${ }^{2}$ Department of Pediatrics, Yamagata Prefectural Central Hospital, Yamagata, Japan and ${ }^{3}$ Advanced Molecular Epidemiology Research Institute, Yamagata University School of Medicine, Yamagata, Japan 


\section{SUBJECTS AND METHODS}

Subjects

The subjects were recruited from among the Japanese full-term and breast-fed neonates born at Yamagata Prefectural Central Hospital from July 1, 2008 to June 30, 2010. We excluded neonates who had factors that would affect the level of serum bilirubin, such as hemolytic anemia, neonatal asphyxia, maternal diabetes, congenital heart or intestine malformation, infections and parenteral fluid therapies. We finally studied the 401 neonates who were exclusively breastfed without supplementation of formula before developing hyperbilirubinemia. Main characteristics of the 401 neonates were as follows: male-to-female ratio, 221:180, gestational age, $39.5 \pm 1.2$ (mean \pm s.d.) weeks; and birth weight, $3080 \pm 325$ (mean \pm s.d.) group. Neonates with severe hyperbilirubinemia were treated with phototherapy according to the criteria. Medical records including gestational age, birth weight, body weight loss [(birth body weight - daily body weight)/birth body weight $\times 100 \%$ ], total serum bilirubin levels and peak bilirubin levels before phototherapy were reviewed. The Ethics Committee of the Yamagata University School of Medicine approved this study. After informed consent was obtained from the parents, genomic DNA was isolated from spare dried blood spots from neonatal screening cards, as described previously. ${ }^{10}$

The 401 neonates were classified into group A (neonates showing $<10 \%$ body weight loss during the neonatal period) and group B (neonates showing $10 \%$ or greater body weight loss during the neonatal period) (Table 1).

Assessment of serum bilirubin level and criteria for phototherapy Serum bilirubin of the neonates was assessed with a Jaundice Meter (model 103; Minolta, Osaka, Japan) once a day, at the same time, during hospitalization or at least during the first week of life. When the reading of the transcutaneous bilirubinometer reached the criterion for further evaluation, the total serum bilirubin concentration was measured. Phototherapy was initiated if the measured bilirubin level exceeded the criterion as follows: $171 \mu \mathrm{moll}^{-1}$ at day $1,239 \mu \mathrm{moll}^{-1}$ at day $2,274 \mu \mathrm{moll} 1^{-1}$ at day 3 , $291 \mu \mathrm{moll}^{-1}$ at day $4,308 \mu \mathrm{moll}^{-1}$ at day 5 and $342 \mu \mathrm{moll} 1^{-1}$ at day 6 .

\section{UGT1A1 gene analysis}

The $(\mathrm{TA})_{7}$ mutation in the promoter and G71R mutation in exon 1 of the UGT1A1 gene were analyzed by fragment analysis ${ }^{11}$ and TaqMan Drug Metabolism Genotyping Assay (c 559715 20) (PE Applied Biosystems, Foster City, CA, USA), respectively.

\section{Statistical analysis}

$\mathrm{R}$ statistical package 2.11.1 for Windows was used for statistical analysis. Differences in the distribution of several categorical and continuous variables between A and B groups were analyzed using the $\chi^{2}$-test and Mann-Whitney $U$-test where appropriate. Simple linear regression and the Cochran-Armitage trend test were used to assess whether the peak serum bilirubin level and incidence of hyperbilirubinemia increased with numbers of non wild-type alleles in genotypes, respectively. Multivariate-adjusted odds ratios (ORs) and

Table 1 Demographic characteristics of the 401 infants enrolled in this study ${ }^{a}$

\begin{tabular}{lccr}
\hline Characteristics & Group A & Group B & P \\
\hline$N$ & 299 & 102 & \\
Male & $169(56.5)$ & $52(51.0)$ & 0.331 \\
Cesarean delivery & $43(14.4)$ & $27(26.5)$ & 0.005 \\
GA (weeks), mean \pm s.d. & $39.7 \pm 1.2$ & $39.2 \pm 1.3$ & 0.005 \\
BW (g), mean \pm s.d. & $3082 \pm 318$ & $3076 \pm 351$ & 0.573 \\
Maximal body weight loss (\%), mean \pm s.d. & $7.2 \pm 1.8$ & $11.8 \pm 1.3$ & $<0.001$ \\
Peak bilirubin level (mg dl ${ }^{-1}$ ), mean \pm s.d. & $11.9 \pm 2.5$ & $12.4 \pm 3.0$ & 0.046 \\
Hyperbilirubinemia & $35(11.7)$ & $21(20.6)$ & 0.025 \\
\hline
\end{tabular}

Abbreviations: BW, birth weight; GA, gestational age.

a Data are expressed as $n(\%)$ unless otherwise noted.

bMaximal body weight loss was calculated as maximal body weight loss in comparison with

body weight divided by birth body weight.
95\% confidence intervals (CIs) of hyperbilirubinemia were estimated for genetic polymorphisms of the UGT1A1 gene and various risk factors selected by univariate analysis using multiple logistic regression analysis. Subset analysis was carried out to evaluate the potential synergistic effect between G71R mutation of the UGT1A1 gene and various levels of maximal body weight loss. A test of interaction was performed by adding a product term to the model. All tests were two-sided. A $P$-value $<0.05$ was considered significant.

\section{RESULTS}

A total of 401 neonates were enrolled in the study, and were classified into two groups. Groups A and B included neonates showing a less than $10 \%$ body weight loss and $10 \%$ or greater body weight loss during the neonatal period, respectively. As shown in Table 1, 56 neonates $(14 \%)$ showed hyperbilirubinemia and required phototherapy. The neonates of group B had a significantly higher peak bilirubin level and incidence of hyperbilirubinemia, higher frequency of cesarean delivery, and shorter gestational period. Sex and body weight at birth were not significantly different between the two groups.

UGT1A1 genotyping of the 401 neonates showed that gene frequencies of $\mathrm{G} 71 \mathrm{R}$ and $(\mathrm{TA})_{7}$ mutations were 0.18 and 0.13 , respectively. As shown in Table 2, the numbers of neonates carrying the $(\mathrm{TA})_{7}$ mutation were not significantly different between the two groups, but neonates carrying the G71R mutation were more frequently detected in group A.

The peak bilirubin level and incidence of hyperbilirubinemia were higher in the neonates of group B compared with those of group A (Table 1). However, significant increases in the peak bilirubin level and incidence of hyperbilirubinemia were only noted in the neonates carrying heterozygous G71R mutation or homozygous (TA) 6 (wildtype) of the promoter of group B (Table 3). The gene dose effect for G71R mutation was not confirmed, possibly because of the small sample size to carry out statistical tests of the neonates with homozygous G71R mutation. $(\mathrm{TA})_{7}$ mutation had no increasing effect on the peak bilirubin level and incidence of hyperbilirubinemia.

Multivariate regression analysis for neonatal hyperbilirubinemia revealed that only maximal body weight loss was an independent risk factor for the development of neonatal hyperbilirubinemia with adjusted OR (95\% CI) of $1.25(1.11-1.41)$ (Table 4). The G71R mutation was not a significant independent risk factor for neonatal hyperbilirubinemia. On the other hand, heterozygous $(\mathrm{TA})_{7}$ mutation and cesarean delivery rather significantly decreased the risk of neonatal hyperbilirubinemia.

Maximal body weight loss was the only independent risk factor for the development of neonatal hyperbilirubinemia, and the association

\section{Table 2 Results of UGT1A1 genetic polymorphisms in A and B} groups $^{\mathrm{a}}$

\begin{tabular}{lccc}
\hline UGT1A1 genotypes & Group $A(\mathrm{~N}=299)$ & Group $B(\mathrm{~N}=102)$ & $\mathrm{P}$ \\
\hline $\begin{array}{l}\text { Coding region } 211 \\
\text { G/G }\end{array}$ & $188(62.9)$ & $79(77.5)$ & 0.026 \\
G/A & $105(35.1)$ & $22(21.6)$ & \\
A/A & $6(2.0)$ & $1(0.9)$ & \\
& & & \\
Promoter & & & \\
$(\mathrm{TA})_{6} /(\mathrm{TA})_{6}$ & $234(78.3)$ & $69(67.6)$ & \\
$(\mathrm{TA})_{6} /(\mathrm{TA})_{7}$ & $59(19.7)$ & $29(28.4)$ & \\
$(\mathrm{TA})_{7} /(\mathrm{TA})_{7}$ & $6(2.0)$ & $4(3.9)$ & \\
\hline
\end{tabular}




Table 3 Correlations of the peak serum bilirubin level and incidence of hyperbilirubinemia in group A versus group B with different UGT1A1 genotypes

\begin{tabular}{|c|c|c|c|c|c|c|}
\hline \multirow{2}{*}{ UGT1A1 genotypes (case number) } & \multicolumn{3}{|c|}{ Peak serum bilirubin level } & \multicolumn{3}{|c|}{ Incidence of hyperbilirubinemia } \\
\hline & $A$, mean \pm s.d. & $B$, mean \pm s.d. & $P$ & $A, n(\%)$ & $B, n(\%)$ & $P$ \\
\hline \multicolumn{7}{|l|}{ Coding region 211} \\
\hline G/G (267) & $11.7 \pm 2.5$ & $11.8 \pm 3.1$ & 0.371 & 21/188 (11.2) & $10 / 79$ (12.7) & 0.729 \\
\hline $\mathrm{G} / \mathrm{A}(127)$ & $12.2 \pm 2.6$ & $14.1 \pm 2.0$ & 0.001 & $13 / 105(12.4)$ & $11 / 22(50.0)$ & $4.2 \times 10^{-5}$ \\
\hline $\mathrm{A} / \mathrm{A}(7)$ & $12.9 \pm 2.4$ & - & - & $1 / 6(16.7)$ & $0 / 1(0)$ & - \\
\hline$P$ for trend test & 0.067 & 0.002 & & 0.657 & $<0.001$ & \\
\hline \multicolumn{7}{|l|}{ Promoter } \\
\hline$(\mathrm{TA})_{6} /(\mathrm{TA})_{6}(303)$ & $12.1 \pm 2.4$ & $12.8 \pm 3.2$ & 0.009 & 29/234 (12.4) & 20/69 (29.0) & 0.001 \\
\hline$(\mathrm{TA})_{6} /(\mathrm{TA})_{7}(88)$ & $11.2 \pm 2.5$ & $11.6 \pm 2.5$ & 0.394 & $6 / 59(10.2)$ & $1 / 29(3.4)$ & 0.273 \\
\hline$(\mathrm{TA})_{7} /(\mathrm{TA})_{7}(10)$ & $11.6 \pm 3.7$ & $10.4 \pm 2.3$ & 0.454 & $0 / 6(0)$ & $0 / 4(0)$ & - \\
\hline$P$ for trend test & 0.021 & 0.027 & & 0.376 & 0.003 & \\
\hline
\end{tabular}

Table 4 Multivariate regression analysis of neonatal hyperbilirubinemia

\begin{tabular}{|c|c|c|}
\hline Variable & Adjusted OR (95\% Cl) & $P$ \\
\hline \multicolumn{3}{|l|}{ Sex of baby } \\
\hline Female & Referent & \\
\hline Male & $1.26(0.69-2.31)$ & 0.459 \\
\hline GA (weeks) & $0.80(0.59-1.07)$ & 0.134 \\
\hline \multicolumn{3}{|l|}{ Cesarean delivery } \\
\hline No & Referent & \\
\hline Yes & $0.27(0.10-0.73)$ & 0.010 \\
\hline BW at birth & $1.00(0.998-1.001)$ & 0.288 \\
\hline Maximal body weight loss (\%) & $1.25(1.11-1.41)$ & $<0.001$ \\
\hline \multicolumn{3}{|l|}{ UGT1A1 gene-coding region 211} \\
\hline $\mathrm{G} / \mathrm{G}$ & Referent & \\
\hline $\mathrm{G} / \mathrm{A}$ & $1.76(0.94-3.28)$ & 0.075 \\
\hline$A / A$ & $1.18(0.13-10.84)$ & 0.881 \\
\hline \multicolumn{3}{|l|}{ UGT1A1 gene promoter } \\
\hline$(\mathrm{TA})_{6} /(\mathrm{TA})_{6}$ & Referent & \\
\hline$(\mathrm{TA})_{6} /(\mathrm{TA})_{7}$ & $0.37(0.15-0.89)$ & 0.027 \\
\hline$(\mathrm{TA})_{7} /(\mathrm{TA})_{7}$ & - & - \\
\hline
\end{tabular}

Abbreviations: BW, birth weight; $\mathrm{Cl}$, confidence interval; GA, gestational age; OR, odds ratio.

between the degree of maximal body weight loss and the G71R genotype of the UGT1A1 gene was studied. Subgroup analysis revealed that the G71R mutation was a significant risk factor for the development of neonatal hyperbilirubinemia in the neonates with $5.0 \%$ or greater maximal body weight loss (Table 5 ). The influence of the G71R mutation increased in parallel with the degree of maximal body weight loss. Neonates who carried heterozygous G71R mutation of the UGT1A1 gene showed a relative risk (OR: 2.23; 95\% CI: $1.21-$ 4.11) for the development of neonatal hyperbilirubinemia in infants with a $5.0 \%$ or greater maximal body weight loss; whereas the corresponding OR $(95 \% \mathrm{CI})$ was $2.75(1.35-5.62)$ in infants with a $7.5 \%$ or greater maximal body weight loss and the OR $(95 \% \mathrm{CI})$ was $6.2(2.08-18.50)$ in infants with a $10.0 \%$ or greater maximal body weight loss. Data on homozygous G71R mutation did not show significant difference possibly because of the small size of samples.
Table 5 Subgroup analysis of UGT1A1 gene-coding region 211 genotypes and various levels of maximal body weight loss

\begin{tabular}{|c|c|c|c|c|c|}
\hline \multirow{2}{*}{$\begin{array}{l}\text { Stratifying } \\
\text { variable }\end{array}$} & \multicolumn{5}{|c|}{$\begin{array}{c}\text { UGT1A1 gene-coding region } 211 . \\
\text { Adjusted OR }(95 \% \mathrm{Cl})^{\mathrm{a}}\end{array}$} \\
\hline & $G / G$ & $G / A$ & $P$ & $A / A$ & $P$ \\
\hline \multicolumn{6}{|c|}{ Maximal body weight loss } \\
\hline$<5.0 \%$ & Referent & - & - & - & - \\
\hline$\geqslant 5.0 \%$ & Referent & $2.23(1.21-4.11)$ & 0.010 & $1.86(0.2-17.41)$ & 0.588 \\
\hline \multicolumn{6}{|c|}{ Maximal body weight loss } \\
\hline$<7.5 \%$ & Referent & $1.00(0.30-3.34)$ & 0.990 & - & - \\
\hline$\geqslant 7.5 \%$ & Referent & $2.75(1.35-5.62)$ & 0.005 & $1.73(0.18-16.54)$ & 0.634 \\
\hline \multicolumn{6}{|c|}{ Maximal body weight loss } \\
\hline$<10 \%$ & Referent & $1.27(0.60-2.71)$ & 0.534 & $1.96(0.20-18.88)$ & 0.560 \\
\hline$\geqslant 10 \%$ & Referent & $6.20(2.08-18.50)$ & 0.0011 & - & - \\
\hline
\end{tabular}

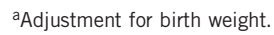

\section{DISCUSSION}

We studied the association of G71R and $(\mathrm{TA})_{7}$ mutations and hyperbilirubinemia of breast-fed Japanese neonates, and found that maximal body weight loss during the neonatal period is the only independent risk factor for the development of neonatal hyperbilirubinemia, and G71R mutation is a significant risk factor only in neonates with a $5.0 \%$ or greater maximal body weight loss.

Neonatal hyperbilirubinemia is a physiological phenomenon, but its severity is also affected by genetic factors. We previously reported that G71R mutation of the UGT1A1 gene was prevalent in Japanese, Koreans and Chinese and was associated with neonatal hyperbilirubinemia., 4 However, half of the Japanese neonates with severe hyperbilirubinemia did not carry the G71R mutation. To identify other predisposing factors of neonatal hyperbilirubinemia, we studied the relation with the enhancer sequence $(-3483 /-3194)$ (phenobarbital response enhancer 
module) of the UGT1A1 gene, polymorphic (GT)n repeats in the promoter region of the hemeoxygenase-1 (a rate-limiting enzyme in heme metabolism) gene, and the composition of fetal hemoglobin (a probable major source of bilirubin in neonates). ${ }^{12,13}$ However, we could not identify any other significant genetic risk factors except the G71R mutation of UGT1A1. Recently, a significant association of G71R mutation with hyperbilirubinemia in breast-fed neonates was reported. ${ }^{7,8}$ We classified the full-term breast-fed neonates into two groups depending on the degree of maximal body weight loss. Cesarean delivery and a short pregnancy period were frequently observed in neonates of group B. Cesarean delivery may cause a delayed onset of lactation, leading to an increase in the maximal body weight loss. ${ }^{14}$ However, multivariate regression analysis revealed that cesarean delivery and heterozygous $(\mathrm{TA})_{6}$ mutation rather decreased the risk of neonatal hyperbilirubinemia. Chang et al. ${ }^{8}$ reported that breast-fed neonates with vaginal delivery have a high risk for the development of hyperbilirubinemia. They speculated that vaginal delivery might be associated with oxytocin usage, vacuum extraction and cephalohematoma. Oxytocin exposure is a risk factor for hyperbilirubinemia, which may have some direct effect on neonatal bilirubin metabolism. ${ }^{15,16}$ The association of heterozygous (TA) 6 mutation with a decrease in the risk for hyperbilirubinemia is unknown.

Our findings indicate that maximal body weight loss, that is, inadequate feeding, is an independent risk factor for the development of neonatal hyperbilirubinemia in breast-fed neonates, and the effect of G71R on neonatal hyperbilirubinemia is significant only in infants under inadequate feeding. Chang et al. ${ }^{17}$ reported that neonates with greater weight loss percentage were associated with hyperbilirubinemia. An $8 \%$ or greater weight loss after $48 \mathrm{~h}$ and an $11 \%$ or greater weight loss after $72 \mathrm{~h}$ significantly increased a risk for hyperbilirubinemia. Ishihara et al. ${ }^{18}$ demonstrated that healthy individuals with UGT1A1 mutation presented with a significant increase in serum bilirubin levels compared with individuals without any mutation after caloric restriction. Inadequate feeding of breast-fed neonates may increase intestinal bilirubin absorption, impair hepatic conjugation and or excretion of bilirubin because of energy deficiency, and cause hyperbilirubinemia in neonates carrying the G71R mutation. The American Academy of Pediatrics recommends that clinicians should advise mothers to nurse their infants at least 8-12 times per day for the first several days. ${ }^{19}$ Our data suggest that adequate feeding could overcome the genetic predisposing factor (G71R mutation of UGT1A1) of neonatal hyperbilirubinemia even in breast-fed infants.
1 Halamek., L. P. \& Stevenson, D. K. In Neonatal-Perinatal Medicine. (eds Fanaroff, A. A. \& Martin, R. J.) 1345-1389 (Mosby-Year Book, St. Louis, 1997).

2 Kawade, N. \& Onishi, S. The prenatal and postnatal development of UDPglucuronyltransferase activity towards bilirubin and the effect of premature birth on this activity in the human liver. Biochem. J 196, 257-260 (1981).

3 Beutler, E., Gelbart, T. \& Demina, A. Racial variability in the UDP-glucuronosyltransferase 1 (UGT1A1) promoter: a balanced polymorphism for regulation of bilirubin metabolism? Proc. Natl Acad. Sci. USA 95, 8170-8174 (1998).

4 Akaba, K., Kimura, T., Sasaki, A., Tanabe, S., Ikegami, T., Hashimoto, M. et al. Neonatal hyperbilirubinemia and mutation of the bilirubin uridine diphosphateglucuronosyltransferase gene: a common missense mutation among Japanese, Koreans and Chinese. Biochem. Mol. Biol. Int. 46, 21-26 (1998).

5 Akaba, K., Kimura, T., Sasaki, A., Tanabe, S., Wakabayashi, T., Hiroi, M. et al. Neonatal hyperbilirubinemia and a common mutation of the bilirubin uridine diphosphateglucuronosyltransferase gene in Japanese. J. Hum. Genet. 44, 22-25 (1999).

6 Long, J., Zhang, S., Fang, X., Luo, Y. \& Liu, J. Association of neonatal hyperbilirubinemia with uridine diphosphate-glucuronosyltransferase $1 \mathrm{~A} 1$ gene polymorphisms: meta-analysis. Pediatr. Int. 53, 530-540 (2011).

7 Chou., H. C., Chen, M. H., Yang, H. I., Su, Y. N., Hsieh, W. S., Chen, C. Y. et al. 211G to $A$ variation of UDP-glucuronosyl transferase $1 \mathrm{~A} 1$ gene and neonatal greastfeeding jaundice. Pediatr. Res. 69, 170-174 (2011).

8 Chang, P. F., Lin, Y. C., Liu, K., Yeh, S. J. \& Ni, Y. H. Risk of hyperbilirubinemia in breast-fed Infants. J. Pediatr. 159, 561-565 (2011).

9 Gourley, G. R. Breast-feeding neonatal jaundice and kernicterus. Semin. Neonatol. 7, 135-141 (2002).

10 Jinks, D. C., Minter, M., Tarver, D. A., Vanderford, M., Hejtmancik, J. F. \& McCabe, E. R. Molecular genetic diagnosis of sickle cell disease using dried blood specimens on blotters used for neonatescreening. Hum. Genet. 81, 363-366 (1989).

11 Baudhuin, L. M., Highsmith, W. E., Skierka, J., Holtegaard, L., Moore, B. E. \& O'Kane, D. J. Comparison of three methods for genotyping the UGT1A1 (TA)n repeat polymorphism. Clin. Biochem. 40, 710-717 (2007).

12 Kanai, M., Kijima, K., Shirahata, E., Sasaki, A., Akaba, K., Umetsu, K. et al. Neonatal hyperbilirubinemia and the bilirubin uridine diphosphate-glucuronosyltransferase gene: the common $-3263 T>G$ mutation of phenobarbital response enhancer module is not associated with the neonatal hyperbilirubinemia in Japanese. Pediatr. Int 47, 137-141 (2005).

13 Kanai, M., Akaba, K., Sasaki, A., Sato, M., Harano, T., Shibahara, S. et al. Neonatal hyperbilirubinemia in Japanese neonates: analysis of the heme oxygenase-1 gene and fetal hemoglobin composition in cord blood. Pediatr. Res. 54, 165-171 (2003).

14 Dewey, K. G., Nommsen-Rivers, L. A., Heinig, M. J. \& Cohen, R. J. Risk factors for suboptimal infant breastfeeding behavior, delayed onset of lactation, and excess neonatal weight loss. Pediatrics 112, 607-619 (2003).

15 Keren, R., Bhutani, V. K., Luan, X., Nihtianova, S., Cnaan, A. \& Schwartz, J. S. Identifying newborns at risk of significant hyperbilirubinaemia: a comparison of two recommended approaches. Arch. Dis. Child 90, 415-421 (2005).

16 Calder, A. A., Ounsted, M. K., Moar, V. A. \& Turnbull, A. C. Increased bilirubin levels in neonates after induction of labour by intravenous prostaglandin E2 or oxytocin. Lancet 2, 1339-1342 (1974).

17 Chang, R. J., Chou, H. C., Chang, Y. H., Chen, M. H., Chen, C. Y., Hsieh, W. S. et al. Weight loss percentage prediction of subsequent neonatal hyperbilirubinemia in exclusively breastfed neonates. Pediatr. Neonatol. 53, 41-44 (2012).

18 Ishihara, T., Kaito, M., Takeuchi, K., Gabazza, E. C., Tanaka, Y., Higuchi, K. et al. Role of UGT1A1 mutation in fasting hyperbilirubinemia. J. Gastroenterol. Hepatol. 16 678-682 (2001).

19 American Academy of Pediatrics. Subcommittee on Hyperbilirubinemia. Management of hyperbilirubinemia in the newborn infant 35 or more weeks of gestation. Pediatrics 114, 297-316 (2004). 